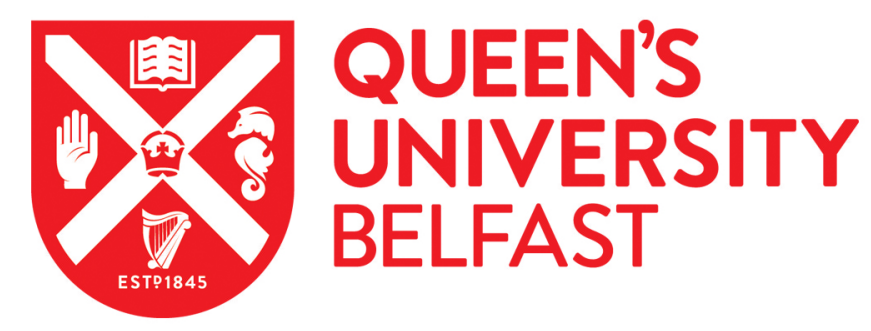

\title{
First principles simulation of damage to solvated nucleotides due to shock waves
}

Fraile, A., Smyth, M., Kohanoff, J., \& Solov'yov, A. V. (2019). First principles simulation of damage to solvated nucleotides due to shock waves. Journal of Chemical Physics, 150(1), [015101].

https://doi.org/10.1063/1.5028451

Published in:

Journal of Chemical Physics

Document Version:

Peer reviewed version

Queen's University Belfast - Research Portal:

Link to publication record in Queen's University Belfast Research Portal

Publisher rights

(c) 2019 The Authors. This work is made available online in accordance with the publisher's policies. Please refer to any applicable terms of use of the publisher.

\section{General rights}

Copyright for the publications made accessible via the Queen's University Belfast Research Portal is retained by the author(s) and / or other copyright owners and it is a condition of accessing these publications that users recognise and abide by the legal requirements associated with these rights.

Take down policy

The Research Portal is Queen's institutional repository that provides access to Queen's research output. Every effort has been made to ensure that content in the Research Portal does not infringe any person's rights, or applicable UK laws. If you discover content in the Research Portal that you believe breaches copyright or violates any law, please contact openaccess@qub.ac.uk. 


\title{
First principles Simulation of Damage to Solvated Nucleotides due to Shock Waves
}

\author{
Alberto Fraile, ${ }^{*}$ Maeve Smyth,$^{\dagger}$ and Jorge Kohanoff ${ }^{\ddagger}$ \\ Atomistic Simulation Centre, Queen's University Belfast, \\ Belfast BTr 1NN, Northern Ireland, United Kingdom \\ Andrey V. Solov'yov ${ }^{\S}$ \\ MBN Research Center, Altenhöferallee 3, D-60438 Frankfurt am Main, Germany
}

\begin{abstract}
We present a first-principles molecular dynamics study of the effect of shock waves (SW) propagating in a model biological medium. We find that the SW can cause chemical modifications through varied and complex mechanisms, in particular phosphate-sugar and sugar-base bond breaks. In addition, the SW promotes the dissociation of water molecules thus enhancing the ionic strength of the medium. Freed protons can hydrolyze base and sugar rings previously opened by the shock. However, many of these events are only temporary, and bonds reform rapidly. Irreversible damage is observed for pressures above 15-20 GPa. These results are important to gain a better understanding of the microscopic damage mechanisms underlying cosmic-ray irradiation in space and ion-beam cancer therapy.
\end{abstract}

\section{INTRODUCTION}

At $\mathrm{MeV}$ energies, ions deposit their energy in the biological tissue by ionizing the medium and generatinging radicals and low-energy secondary electrons (LEE). [14] Until the seminal work of Boudaiffa et al., [5] $\mathrm{OH}^{\bullet}$ radicals were deemed to be the main source of indirect damage to DNA. Since then, attention has turned also to the effect of LEE [6-13] and, to a lesser extent, to that of radicals. [14] A third aspect, that has been largely ignored until recently, is the radial shock wave (SW) driven by the forces generated along the track, especially in the case of swift heavy ions. The energy carried by the SW is distributed around the system and can produce lesions to functionally important structures such as DNA, proteins and membranes.[15-17]

Ref. [15] studied the thermo-mechanical effects in the vicinity of the ion-tracks by means of the thermal spike model. This model does not account for the dynamics of the medium, therefore not including the possibility of SWs. The SW effect was suggested and described within the framework of the conventional hydrodynamics in Ref. [16] and was further demonstrated by means of classical molecular dynamics (MD) simulations. These effects could be relevant in ion-beam radiotherapies, cosmic radiation shielding and space medicine. In fact, a recently proposed model $[16,17]$ suggested that a sizeable fraction of the damage due to ionizing radiation could be induced by the SW, especially for heavier ions.

\footnotetext{
* Present address: Faculty of Electrical Engineering, Czech Technical University in Prague, Karlovo náměstí 13, 121 35, Prague 2, Czech Republic

$\dagger$ Present address: Velindre Cancer Centre, Velindre Road, Cardiff, CF14 2TL, United Kingdom

¥ j.kohanoff@qub.ac.uk; Corresponding author

$\S$ Also at: A.F.Ioffe Physical-Technical Institute, Politechnicheskaya Str. 26, 194021 St. Petersburg, Russia and St. Petersburg State Polytechnical University, Politechnicheskaya 29, 195251 St. Petersburg, Russia; E-mail: solovyov@mbnresearch.com
}

Shock waves induced by ion irradiation are a consequence of a localized and fast energy transfer from the ions to the medium, in the form of ionization. In the vicinity of the Bragg peak the average kinetic energy of the secondary electrons produced by such ionization is about $45 \mathrm{eV}$.[18, 19] These secondary electrons transport the energy deposited by the ion less than $1.5 \mathrm{~nm}$ around its path in some tens of femtoseconds, and there is no other mechanism which could dissipate this energy deposited so quickly. Indeed, the molecular diffusion mechanism is too slow and the production of more energetic $\delta$-electrons, which could carry the energy further away vanishes in the Bragg peak region.[20, 21] Therefore, high pressures are expected to develop inside a narrow cylinder around the ion track. In reference [16] it was demonstrated that the ratio of pressures between the inside and the outside of this narrow cylinder is larger than $(\gamma+1) /(\gamma-1)$ with $\gamma=C_{p} / C_{v}=1.222$, thus fulfilling the conditions for a strong cylindrical explosion.[22] This creates a discontinuity in the initial conditions for pressure that starts propagating in the radial direction away from the ion path. This propagation also features discontinuities in density and collective flow velocity and is referred to as a shock wave.[22, 23]

Apart from the direct action of the SW, it has been proposed that the SW may also influence other damage mechanisms, e.g. by promoting the dissociation of certain bonds that have been weakened due to electron attachment to antibonding orbitals, or by facilitating the diffusion of radicals towards DNA. These mechanisms have been further investigated in more recent works. [24-27]

The onset of shock waves (or blast waves) as a result of concentrated and sudden energy deposition has been studied both theoretically and experimentally since the early 1940s.[23, 28-32] Analytical solutions for spherical shock waves where derived independently in [29-31, 33]. These solutions are comprehensively presented in references $[22,23]$. Following the derivation for the spherical case, the hydrodynamic equations for the evolution of cylindrical shock waves resulting from the energy de- 
position around ion paths in liquid water were solved in reference [16]. Later on, several molecular dynamics simulations of the dynamics of liquid water following energy deposition in the vicinity of ion paths confirmed the expected behavior of the theoretically predicted shock waves.[25, 26, 34, 35]

However, in none of the papers cited above the ion induced SW effect was studied by ab initio quantum mechanical methods. Although the entire span of the effect on the temporal and spatial scales is a limiting factor for the utilization of quantum approaches, these methods are capable of providing very important nanoscopic insights and quantitative descriptions of the molecular damage caused to DNA, water, and other biologically relevant molecules. This damage to the medium, which does not need to be biological, arises under the conditions generated by their irradiation with ions, in particular in the vicinity of the Bragg peak. This knowledge is relevant to several applications, including medical, e.g. ion-beam cancer therapy, and space electronics. The applicability of first-principles molecular dynamics simulation (FPMD) to the description of molecular damage is simply related to the fact the size of the volume in which the damage occurs and the duration of the quantum processes leading to molecular dissociation and the formation of new bonds are accessible to FPMD with present-day computational resources.

The relevance of these simulations to the aforementioned ion-induced SW effect should be established through the relevant choice of the conditions imposed on the medium in the quantum simulation box. These conditions should correspond to those that are well established in the publications cited above through analytical hydrodynamics and statistical mechanical methods as well as by means of classical MD simulations. Consequently, in this work we impose the pressure gradients corresponding to those that arise in classical MD simulations and analytical models, and this is achieved by the choice of relevant particle velocities as will be discussed below. The review of the entire multi-scale approach to the physics of radiation damage by ions can found in Refs. [20] and [36].

While the ion track that initiates the SW can interact directly with DNA, it is statistically more likely that the $\mathrm{SW}$ is generated in the surrounding medium. In the physiological environment DNA appears in the form of chromatin, and it is surrounded by proteins and water containing other biological material and ions. A convenient simplification of such scenario is achieved by considering a pure water environment, and deferring the study of the role of other components to a later time.

Early studies of the propagation of SW in pure water were undertaken because of their importance in underwater explosions, [37] and because of their role in cavitation erosion. $[38,39]$ Interest was renewed with the advent of medical procedures accompanied by SW emission such as extracorporeal SW lithotripsy, [40, 41] intraocular laser microsurgery [42] and short-pulse laser ablation.[43, 44]
Shock compression of water, in particular its composition, has been studied experimentally, [45-48] by ab initio simulations [49-52], and both.[53, 54] It has been shown that the high pressures originated in the SW produce water dissociation through the unimolecular (ionization) mechanism, $\mathrm{H}_{2} \mathrm{O} \rightarrow \mathrm{OH}^{-}+\mathrm{H}^{+}$. [51] Protons generated in these events move through water via classical diffusion mechanisms.

Understanding the influence of a SW in the damage mechanisms of biologically relevant systems is a step up in complexity. Due to its paramount importance in astrobiology, some authors have recently addressed the topic of aminoacid synthesis under shock wave conditions, which is closely related to the present work.[55-58]

In the context of DNA damage, one of the present authors investigated shock wave effects using classical force fields.[17, 24-27] Within this approximation, it was suggested that energy can be transferred to the vibrational degrees of freedom of the medium in which it is absorbed. It was demonstrated that this leads to the breaking of certain bonds, in particular phosphodiester bonds, which are responsible for DNA strand breaks. In addition, it was estimated that only heavier ions that produce a signicant linear energy transfer (LET) are capable of inducing direct strand breaks.

The most unbiased way of accounting for these processes is to describe the interactions at a first-principles level. In previous works we have shown that firstprinciples molecular dynamics simulation (FPMD) is a tool well-suited to study DNA damage under normal (non-SW) conditions.[13] The size of the systems amenable to FPMD simulations, however, is much reduced with respect to classical MD. For this reason, and also for the sake of clarity in the interpretation of the results, we will consider here the smallest DNA fragment that contains the bonds that are relevant for radiation damage, i.e. a nucleotide.

The purpose of the present work is to identify and analyse damage mechanisms in solvated nucleotides under the influence of a shock wave, i.e. out-of-equilibrium and at high pressures and temperatures, using FPMD techniques. We will defer a statistical analysis of the present results to future work.

\section{MODEL AND COMPUTATIONAL DETAILS}

The three main pathways for bond cleavage in nucleotides involve the phosphodiester linkages between the sugar and phosphate at the $3^{\prime}$ and $5^{\prime}$ positions and the $\mathrm{N}$ glycosidic $\mathrm{C}_{1^{\prime}}-\mathrm{N}_{1}$ bond between the sugar and the base (see Fig. 1). Calculations on pyrimidine nucleotides [59] showed that the $\mathrm{C}_{3^{\prime}}-\mathrm{O}_{3^{\prime}}$ bond is significantly easier to cleave than $\mathrm{C}_{5^{\prime}}-\mathrm{O}_{5^{\prime}}$. We have thus focused on the most representative $\mathrm{C}_{1^{\prime}}-\mathrm{N}_{1}$ and $\mathrm{C}_{3^{\prime}}-\mathrm{O}_{3^{\prime}}$.

As a first step we constructed a model system consisting of a solvated thymidine monophosphate (dTMP), using the molecular editor Aten.[60] We then carried 


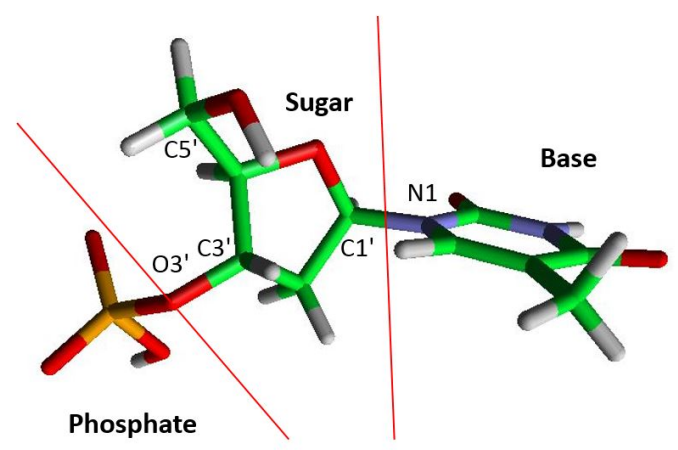

Figure 1. (color online) Nucleotide studied in this work. Hereafter we will refer to its parts as $\mathrm{P}, \mathrm{S}$ and $\mathrm{B}$ for phosphate, sugar and base, respectively. The (red) lines separate the various components of the nucleotide, and indicate the potentially cleaved bonds, phosphodiester and glycosidic.

out a classical molecular dynamics simulation of the periodically replicated box using the simulation package DLPOLY,[61] with the interactions described by the OPLS force field.[62] This sample was equilibrated at $300 \mathrm{~K}$ in the canonical ensemble for $1 \mathrm{~ns}$. Hence, we ran 1 ns of microcanonical MD and extracted a representative reference frame. This was used as the starting point for FPMD simulations using the density functional (DFT) QUICKSTEP module of the CP2K package.[63] The system was equilibrated in the canonical ensemble during 2 ps to reconcile geometric differences between OPLS and DFT. Temperature was maintained at $300 \mathrm{~K}$ using a thermostat, which was then switched off for the SW simulation.

The electronic structure was computed at the DFT level, employing the Gaussian and plane waves (GPW) method in which the Kohn-Sham orbitals are expanded in a Gaussian basis set, while the electrostatic energy and potential are calculated using Fourier transforms. Core electrons were replaced by GTH pseudopotentials, and valence electrons were represented using a triple-zeta plus polarization basis set (TZVP-GTH).[64] The charge density was expanded in plane waves up to an energy cutoff of 250 Ry. We used the PBE exchange-correlation functional [65] supplemented with Grimme's semi-empirical dispersion term (DFT-D2).[66] The nucleotide was deprotonated and the negative charge compensated with a $\mathrm{Na}$ atom elsewhere in the solution, so that the supercell was neutral.

$A b$ initio calculations [67] and FPMD simulations [9] have shown that excess electrons, e.g. secondary electrons generated by ionization of the medium, weaken the phosphodiester bond, which is then more prone to dissociation. While excess electrons tend to localize initially in the nucleobase, [8] they transfer to the C-O phosphodiester bond when the latter is stretched beyond a certain distance,[9] due to the crossing of the HOMO and LUMO energy levels.[67] This stretching can be due either to thermal fluctuations or to the resonant phenomenon known as dissociative electron attachment, in which the excess electron forms a transient negative nucleotide anion that decays via dissociation of the $\mathrm{C}-\mathrm{O}$ bond.[13] Given the relevance in lowering the dissociation barrier, we deemed important to conduct the same SW simulations but in the presence of an excess electron. To this end we used spin-polarized DFT within the PBE approximation [65] to account for the unpaired electron in the simulation cell, and the extra charge was compensated with a uniform positive background.

We used a time step of $0.5 \mathrm{fs}$ and a self-consistency accuracy of $10^{-6}$ Hartree. While shorter time steps of the order of $0.2 \mathrm{fs}$ are advisable under the present conditions, [51] we have checked that even in the most energetic cases considered there is only a minor effect in energies and forces. We consider this sufficient for the present purposes of revealing chemical events like bond breaking and formation that occur in the nucleotide due to the development of the shock. The total time of the simulations was $0.5 \mathrm{ps}$. While chemical equilibration would require longer runs (2 ps for water [51]), this choice was made based on the equilibration of thermodynamic quantities, which occurred within the above time scale.

An intuitive way of simulating a SW is to assign a given velocity to a group of water molecules on one side of the sample and monitor how this perturbation propagates and reaches the dTMP (single shock, SSW). This simulation is run nominally in the NVE ensemble. However, while the energy and volume of the simulation box remain constant, the effective volume of the sample decreases during the compression phase, leaving a vacuum (rarefaction) region behind. A similar approach was used in Ref. [68] to study the effect of shock waves on lipid bilayers. We have implemented this and a related scheme consisting of shocking the sample from both sides (double shock, DSW). These simulations are implemented under periodic boundary conditions ( $\mathrm{PBC})$, so that there is no danger of reflections of the wave. PBC, however, introduce periodic images of the system that could potentially interact with each other. In the present SSW and DSW simulations the propagation of the wave generates a rarified region behind the shock front creating a vacuum between periodic images, which remained during the whole length of the simulations. Therefore, there was no interaction between images.

It is important to remark that neither of the above describes correctly the thermodynamic states of a shocked sample. The relation between pressure and temperature should be described by the Rankine-Hugoniot equations that relate measured kinetic parameters to thermodynamic variables. [69] This can be accomplished using the multi-scale shock technique (MSST).[70, 71] MSST dynamically evolves the cell volume and temperature to maintain the system on both the Rayleigh line $p-p_{0}=$ $u_{s}^{2}\left(V_{0}-V\right) \rho_{0} / V_{0}$ and the shock Hugoniot under conditions of uniaxial strain of the computational cell.[75] Here $u_{s}$ is the shock velocity, $p$ the pressure, $\rho$ the mass density, and $V$ the volume, while the subscript 0 stands for initial values. By regulating the strain rate of the simula- 
tion cell, the $(P, T)$ thermodynamic states accessed during the shock simulation correspond to a steady macroscopic SW. This method presents several advantages, most importantly the requirement of a smaller number of molecules to converge thermodynamic quantities, which is particularly important within the present FPMD context. While MSST still implements PBC, the moving boundary represents the wave front, hence precluding the possibility that the wave interacts with the boundary.

It is not obvious which one of these strategies is closer to the actual experimental situation. On the one hand, under typical irradiation conditions, tracks are spatially well-separated so that the SSW scenario seems more relevant. On the other, the MSST approach is consistent with SW conditions. The DSW scenario is closer to MSST than SSW. We therefore decided to study all three approaches.

Fig. 2 shows a view of the sample before the passage of the SW. The dimensions of the supercell are $50 \times 15 \times 15 \AA^{3}$, containing 843 atoms, (270 water molecules). We modeled a SSW by assigning appropriate initial velocities to an 80 -molecule water slab $(\approx 10 \AA$ in the $x$-direction) to the left of the dTMP. This leaves, for this particular cross section of $15 \times 15 \AA^{2}$, about 70 water molecules between the initial SW front and dTMP (the buffer). We modelled a DSW by assigning velocities as above to 80 -molecule water slabs on both sides of the sample, in opposite directions. These initial velocities were supplemented with thermal velocities in all atoms, extracted from NVT simulations. SSW and DSW fall into the category of non-equilibrium MD simulations (NEMD). The MSST simulations were conducted using the implementation available in CP2K.[63] We carried out SSW, DSW, and MSST simulations with initial velocities between 5 and $10 \mathrm{~km} / \mathrm{s}$.

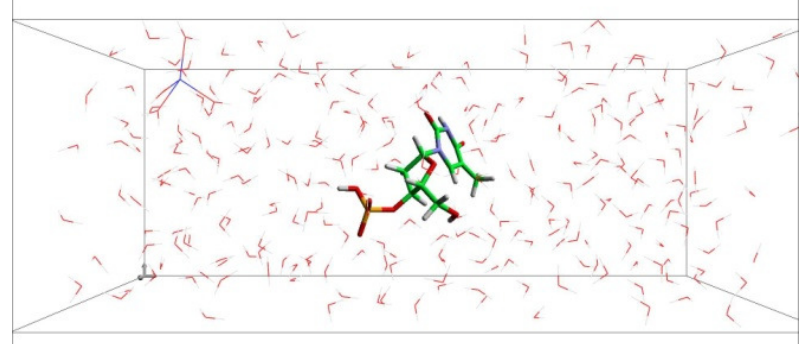

Figure 2. (color online) Model system before the SW compression. Dimensions are $50 \times 15 \times 15 \AA^{3}$ and the number of atoms is 843 ( 270 water molecules). The water molecules are shown as wireframe, while dTMP is shown with thick sticks. A Na atom introduced to compensate the charge can be seen in blue at the top left corner.

It has been argued that compression can cause the closure of the electronic band gap in water, so that it is necessary to include electronic excitations into SW simulations.[72] Electronic conduction, however, requires temperatures above $4000 \mathrm{~K}$ at pressures above $60 \mathrm{GPa}$, and even higher at lower pressures.[72] Therefore, follow- ing Goldman et al., [51] we decided to run the present simulations in the Born-Oppenheimer approximation, which considers ground state electrons. Let us mention here that the simulation of dynamical energy exchanges between electrons and nuclei is an active area of research.[73] Within the present context of MSST simulation of SW, an interesting methodology has been proposed by Reed, which introduces the dynamics of the electronic temperature[74], by assuming that it is homogeneous across the sample.

\section{PHYSICAL PROPERTIES OF THE SHOCK-COMPRESSED SYSTEM}

We first consider the NEMD simulations labelled SSW and DSW. As the SW develops, the water molecules in the shock front collide with those in the buffer initially at $300 \mathrm{~K}$. The result is a time-evolving velocity profile that proceeds towards homogeneity and energy equipartition. To illustrate this process, we show in Fig. 3 the averaged velocity along the $x$ axis, $V_{x}$, as a function of the position in the simulation cell, at different times, for a DSW simulation with an initial velocity of $10 \mathrm{~km} / \mathrm{s}$. It has to be noted that, for such a high initial velocity value, at the end of the simulation the size of the sample has reduced to half its initial value (the simulation cell remains fixed, but a vacuum region develops behind the shocked waters). As expected, the water molecules in the shock front, which started at $10 \mathrm{~km} / \mathrm{s}$, progressively slow down as they exchange energy with the water in the buffer and the nucleotide. These latter increase their velocity at the expenses of the former, as can be observed in the velocity profile at $0.1 \mathrm{ps}$. Clearly, at this time the system is still out of equilibrium, but it is also visible that the velocity profile evolves towards a flat distribution, consistent with thermodynamic equilibrium.

In these NEMD simulations, also physical properties such as pressure and temperature evolve in time towards thermodynamic equilibrium. However, during equilibration these quantities are not uniform across the sample (see Fig. 3 for the velocity), and hence cannot be interpreted as thermodynamic quantities. For this reason we report spatially-averaged quantities only at the end of the runs when the system is closer to equilibrium.

As a consquence of the attenuation of the SW, in the NEMD simulations the effective velocity, $V_{\text {eff }}$, of the wave front reaching the nucleotide is significantly smaller than the initial one, e.g. $4 \mathrm{~km} / \mathrm{s}$ after $150 \mathrm{fs}$ for an initial velocity of $10 \mathrm{~km} / \mathrm{s}$. Clearly, $V_{\text {eff }}$ decreases with increasing thickness of the water buffer. This can also be interpreted as a dependence of $V_{\text {eff }}$ with distance between the ionic track - where the SW is generated - and the nucleotide. In the present sample, initial velocities under $7 \mathrm{~km} / \mathrm{s}$ lead to non-compressive solutions, being $V_{\text {eff }}$ lower than the speed of sound $(1.5 \mathrm{~km} / \mathrm{s}$ in water $)$. To avoid ambiguities, we will report our results in terms of $V_{\text {eff }}$, which we defined as the average velocity of the water molecules rel- 


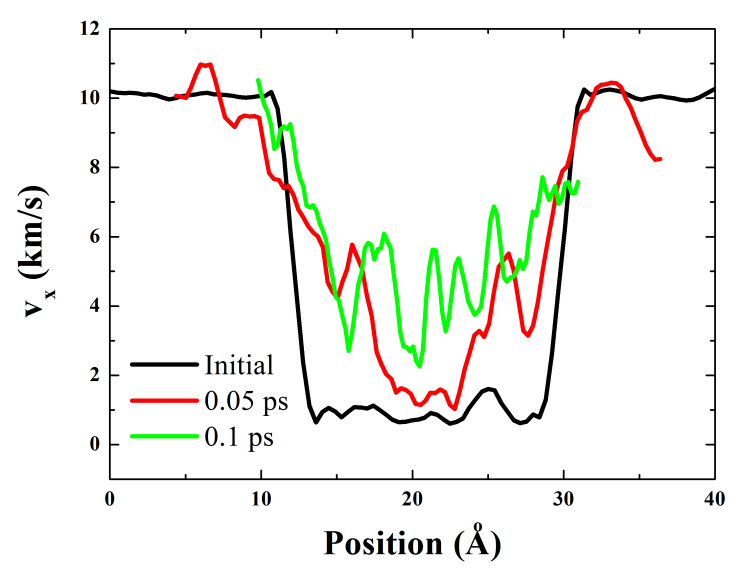

Figure 3. (color online) Time evolution of the velocity profile across the sample, for a double shock (DSW) initiated with a velocity of $10 \mathrm{~km} / \mathrm{s}$. The profile is reported for the initial time (black), after 0.05 ps (red) and after 0.1 ps (green).

ative to dTMP as the wave front reaches the nucleotide.

But before that, we report in Fig. 4 the pressure achieved as a function of $V_{i}$, the shock velocity, obtained from the MSST simulations. We also compare to previous MSST results obtained using[51] and to experimental data. [78] The three sets of values are in very good agreement. Discrepancies between the two sets of MSST data can be due to the use of different functionals and basis sets, or to the presence of the nucleotide.[76] Calculated temperatures are also similar to those of [51] with values around $3000 \mathrm{~K}$ at $10 \mathrm{~km} / \mathrm{s}$. Pressures from DSW nonequilibrium simulations are similar to those in MSST, but those from SSW are much smaller. This is understandable, given that the volume reduction is approximately half that in DSW and MSST simulations. In NEMD simulations, the temperature of the sample is inhomogeneous, and can reach very high values locally, before equilibrating. Equilibrium values are generally lower than those form MSST simuations.

\section{WATER DISSOCIATION AND CHEMICAL REACTIONS}

We now turn our attention to the chemical modifications induced by the passage of the SW. Firstly, it should be noted that the main destructive effect is produced during the rarefaction wave that follows the compression. In Fig. 5 we show a snapshot of the nucleotide during the passage of a SW that reaches dTMP with $V_{\text {eff }} \approx 3 \mathrm{~km} / \mathrm{s}$, corresponding to a SSW with $V_{i}=7 \mathrm{~km} / \mathrm{s}$. The phosphodiester bond is broken, thus suggesting the possibility of strand breaks due to thermo-mechanical effects. In addition, the glycosidic bond between sugar and base is broken. Interestingly, this picture, which is based on a visual inspection of an instantaneous frame, can be quite

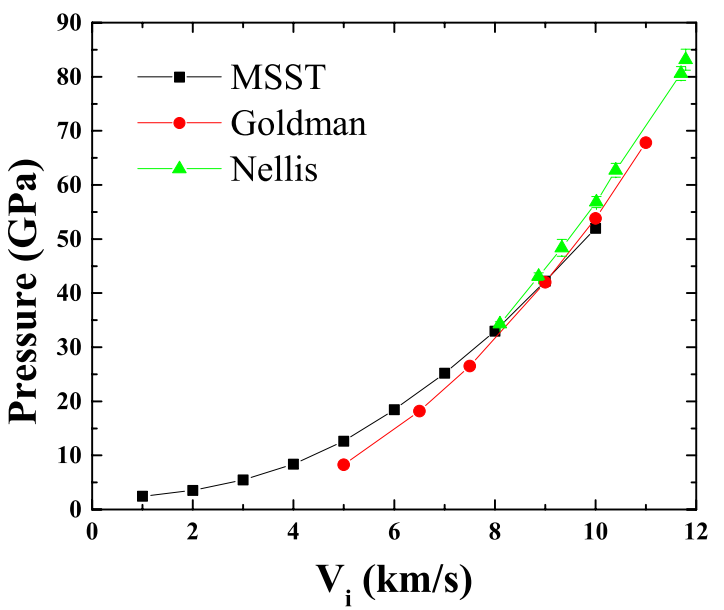

Figure 4. (color online) Pressure vs SW velocity from our MSST simulations (black squares), from the work of Goldman et al. [51] (red circles), and experimental data from Mitchell and Nellis [78] (green triangles).

misleading. In fact, the resulting fragments are reactive species and, unless they are rapidly solvated by water or saturated via proton transfer from the water, they are prone to form bonds again. As a matter of fact, when the simulation is continued, in many cases the original bonds reform, thus exhibiting no apparent long-term damage.

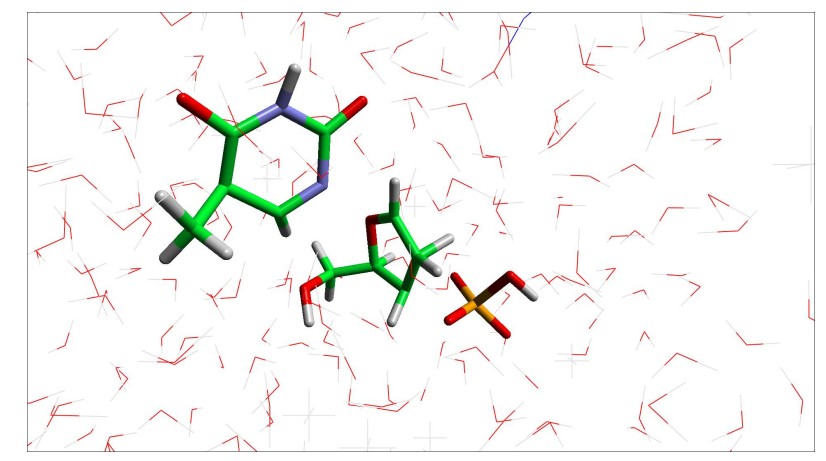

Figure 5. (color online) Nucleotide after the compression created by a SSW with $V_{i}=7 \mathrm{~km} / \mathrm{s}$, corresponding to $V_{\text {eff }} \approx 3$ $\mathrm{km} / \mathrm{s}$. Both, the phosphodiester and the glycosidic bonds are temporarily broken. Inset: Dissociation of water (in $\%$ of $\mathrm{H}$ atoms) as a function of $V_{\text {eff }}$.

Table I summarizes qualitatively the results of bond breaks during our simulations with $V_{\text {eff }}$ ranging from 1 to $4 \mathrm{~km} / \mathrm{s}$, for the neutral case and in the presence of an excess electron. The bonds that break after the SW are denoted as P, S and G for phosphodiester, sugar ring, and glycosidic, respectively. When more than one bond is broken, the order is chronological. Additionally, bonds that break and reform are denoted in parenthesis. The easiest bond to break in dTMP is the phosphodiester bond, which is related to strand breaks in DNA, but the 
Table I. Summary of the SW effect on dTMP. SSW and DSW stand for single and double SW, Label (n) indicates neutral and (ee) negatively charged. The dash means no bond breaks, $\mathrm{P}$ is the phosphodiester bond (strand break), S stands for sugar opening, and $\mathrm{G}$ for glycosidic bond (base excision). Lablels into parenthesis indicate bonds that are broken and quickly reform.

\begin{tabular}{cccccccc}
\hline$V_{\mathrm{i}}(\mathrm{km} / \mathrm{s})$ & $V_{\text {eff }}(\mathrm{km} / \mathrm{s})$ & SSW $(\mathrm{n})$ & SSW $($ ee $)$ & DSW $(\mathrm{n})$ & DSW $($ ee $)$ & MSST $(\mathrm{n})$ & MSST (ee) \\
\hline \hline 5 & 0.3 & - & - & $(\mathrm{S})$ & - & - & - \\
6 & 1.3 & - & - & $\mathrm{P}$ & - & - & $\mathrm{S}$ \\
7 & 1.8 & $(\mathrm{P})$ & $\mathrm{S}+\mathrm{G}$ & $\mathrm{P}$ & $\mathrm{P}$ & $(\mathrm{G})+(\mathrm{S})$ & $(\mathrm{P})+\mathrm{S}$ \\
8 & 2.8 & $\mathrm{P}$ & $\mathrm{P}$ & $\mathrm{P}$ & $\mathrm{P}+\mathrm{S}$ & $(\mathrm{G})+(\mathrm{P})+(\mathrm{S})$ & - \\
10 & 3.7 & $\mathrm{P}+\mathrm{S}$ & $\mathrm{G}$ & $\mathrm{G}+\mathrm{S}+\mathrm{P}$ & $\mathrm{P}$ & $\mathrm{G}+\mathrm{S}+\mathrm{P}$ & $\mathrm{G}+\mathrm{S}+\mathrm{P}$ \\
\hline
\end{tabular}

opening of the sugar ring at the $\mathrm{O}$ atom is also a regular feature. This is to say that the breaking and making of bonds is a dynamical process that depends also on initial conditions. The study of the long-term damage, i.e. which bonds are more likely to remain broken, would require longer simulations and statistical averaging, and was deferred to future work. However, is clear that for very high velocities, i.e. above $10 \mathrm{~km} / \mathrm{s}$, both DSW or MSST indicate a proliferation of broken bonds that, either are not repaired, or form new wrong bonds.

An important consequence of shock compression is an enhanced ionic strength of the medium due to water dissociation. [77]. Recent simulations showed that this process begins at pressures around $12 \mathrm{GPa}$. [53, 54] This generates an abundance of free protons that can react with the biomolecules. Note that the electrical conductivity of shocked water increases rapidly with pressure to reach a plateau between 30-60 GPa.[78] Previous simulations of shocked water showed that proton concentrations can achieve mole fractions of up to $40 \%$ at shock velocities of $10 \mathrm{~km} / \mathrm{s}$.[51] Our simulations exhibit similar trends, with proton concentrations that increase with shock velocity. At variance with this, our interest here is on the chemistry of the nucleotide, which occurs during the development of the shock and not upon equilibration. Under these conditions, the proton concentration is approximately half the equilibrium value. The enhanced proton concentration is likely to play an important role in the chemical modification process, by hydrolyzing certain bonds in the nucleotide. In fact, in some simulations we observed the opening of the sugar ring with intercalation of a proton in a weakened $\mathrm{C}-\mathrm{O}$ bond that was stretched due to the passage of the SW. A similar scenario was observed for the nucleobase.

We first analyze the case if the neutral system, while the effect of excess electrons is deferred to Section V. The minimum effective SW velocity that causes bond breaks in our sample is $V_{\text {eff }} \approx 1.3 \mathrm{~km} / \mathrm{s}$. Fig. 6 shows the state of dTMP after a DSW simulation with $V_{\text {eff }}=4 \mathrm{~km} / \mathrm{s}$, where a $\mathrm{C}-\mathrm{N}$ bond in the nucleobase is broken due to the passage of the SW (dashed blue line).

The strength of the SW determines the strength of the forces stretching the bonds in the nucleotide. It is proportional to the pressure at the wave front, $P$, where it reaches its maximum value.[16] This pressure decreases with the radius of the wave front, $\rho$, as (see supplemental material in [17]):

$$
P=\frac{1}{\gamma+1} \frac{\beta^{4}}{2} \frac{L E T}{\rho^{2}}
$$

where $\gamma=C_{p} / C_{v}$ (heat capacities at constant pressure and volume, which are considered constant), and $\beta=0.86$ is a dimensionless constant (LET is the linear energy transfer defined above in the Introduction). With this equation in mind we can compute the pressures corresponding to different ions used for irradiation.

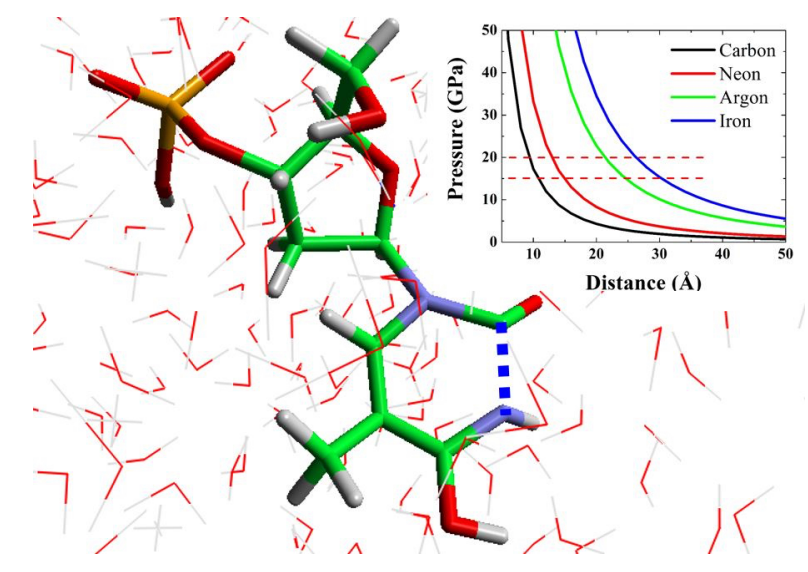

Figure 6. (color online) Nucleotide after a $V_{\text {eff }} \approx 4 \mathrm{~km} / \mathrm{s}$ DSW. The nucleobase has opened at a C-N bond due to the shock (dashed blue line). Inset: Pressure vs distance for four different ions: $\mathrm{C}, \mathrm{Ne}, \mathrm{Ar}$, and $\mathrm{Fe}(\mathrm{LET}=0.9,1.73,4.745$ and $7.195 \mathrm{keV} / \mathrm{nm}$, respectively). At distances larger than $20 \AA$, pressures are large enough to cause irreversible damage only for heavy ions.

The calculated pressure-distance curves are shown in the inset to Fig. 6 for four representative ions, namely C, $\mathrm{Ne}, \mathrm{Ar}$, and Fe. The LETs are 0.9, 1.73, 4.745 and 7.195 $\mathrm{keV} / \mathrm{nm}$, respectively.[17] According to our simulations, the onset of damage occurs at pressures of the order of 20 
GPa, which is indicated by the horizontal dashed line in the inset to Fig. 6. If we consider distances larger than $20 \AA$, then only the heavier ions ( $\mathrm{Ar}, \mathrm{Fe}$ ) will be capable to inflict damage to the nucleotide. The lighter ions $(\mathrm{C}$, $\mathrm{Ne}$, but also protons and $\alpha$ particles) will not deliver sufficient energy to cause serious damage as a consequence of the generated SW.

\section{EXCESS ELECTRONS AND BOND BREAKS}

Simulations in the presence of an excess electron, at relatively low shock velocities, confirmed the localization of the electron in the nucleobase.[8] We do not observe the electron migrating to the sugar-phosphate region as in [9] because, while the SW induces a geometric distortion of the nucleotide, the energy is not deposited coherently into the phosphodiester bond. Therefore, the elongation of the latter is not sufficient for the electronic state located in the sugar-phospate region to become energetically favorable with respect to the state located in the base. On the contrary, at high shock velocities the SW generates geometric distortions in the nucleotide so large that valence-bound states of the excess electron are destabilized. Actually, not much is needed to destabilize such states. We have recently shown that, in solution, the nucleobase cannot stabilize the excess electron in the neutral geometry. It is only after relaxing to anion geometry that the electron localizes, but this geometric distortion is relatively small.[8] In Fig. 7 we show two snapshots taken from a $10 \mathrm{~km} / \mathrm{s}$ MSST simulation, for the system containing an excess electron. The top panel shows the shocked system at $50 \mathrm{fs}$, during the development of the SW. The excess electron is located in the nucleobase, as usual. The spin density that characterizes the excess electron (depicted as a blue surface) is localised in the $\pi$ orbitals around the base. The bottom panel shows the same system at a later time (130 fs), after the compression due to the SW has modified considerably the geometry of the nucleotide. When the deformation is too large, the excess electron is no longer stable in dTMP and it jumps to the water orbitals. This is because when the nucleotide is so severely deformed, the electronic energy level located in the base shifts upwards in energy, ending up above the LUMO of the water orbitals.

Apart from breaking the obvious bonds, the SW can also alter the bonding pattern by breaking some bonds and forming others. Here we present two examples of unusual configurations generated by the passage of the $\mathrm{SW}$. In Fig. 8 one of the $\mathrm{C}-\mathrm{N}$ bonds in the base is broken.

In Fig. 9, the $\mathrm{O}$ in the sugar-phosphate $\mathrm{C}-\mathrm{O}-\mathrm{P}$ bond has been shortcut forming a $\mathrm{P}-\mathrm{C}$ bond between phosphate and sugar, while the sugar ring has opened at a $\mathrm{C}-\mathrm{C}$ bond This unusual pattern is a consequence of the SW breaking the phosphodiester bond, and the $\mathrm{PO}_{4}$ rebonding directly to the $\mathrm{C}$ via the $\mathrm{P}$ atom. While the situations illuatrated above correspond to simulations in

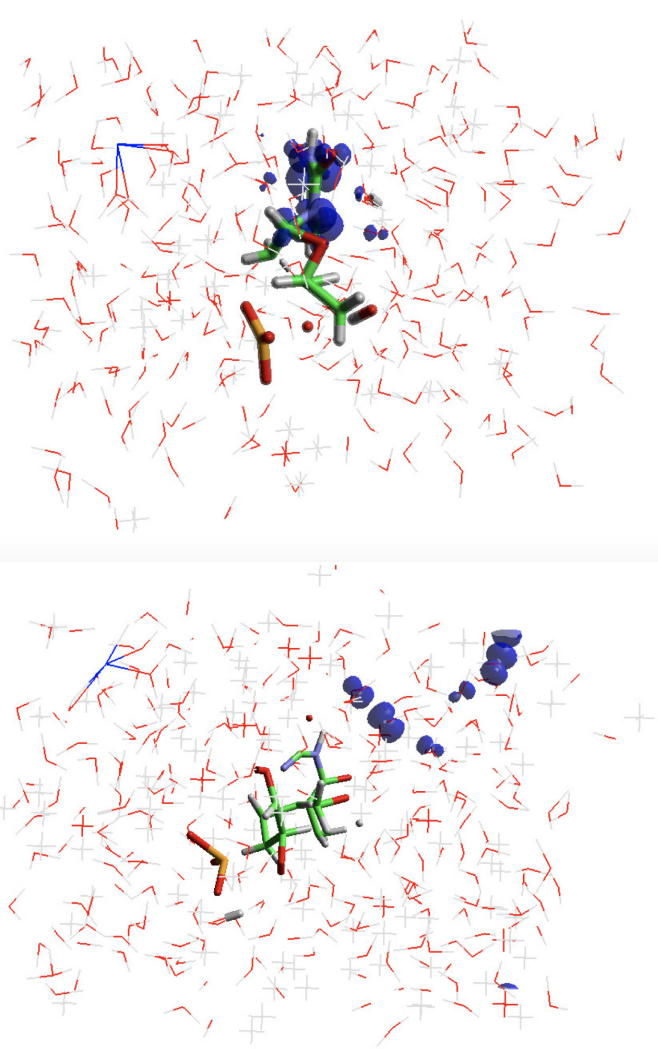

Figure 7. (color online) Top panel: nucleotide after $50 \mathrm{fs}$ in a $V_{i}=10 \mathrm{~km} / \mathrm{s}$ MSST simulation with an excess electron. The phosphodiester $\mathrm{C}-\mathrm{O}$ bond connecting sugar and phosphate is broken and the sugar ring is open, but the base is intact. The spin density that characterizes the excess electron (blue surface) is localised in the $\pi$ orbitals around the base. Bottom panel: same system after 130 fs. Now also the base ring is open. As a consequence, the spin density that characterizes the excess electron (blue surface) has jumped to the water unoccupied orbitals.

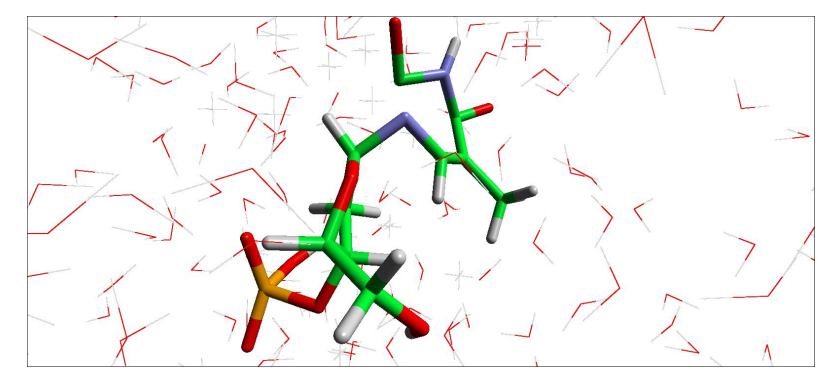

Figure 8. (color online) Nucleotide after a SSW initiated with $V=10 \mathrm{~km} / \mathrm{s}\left(V_{\text {eff }} \approx 4 \mathrm{~km} / \mathrm{s}\right)$, with an excess electron. The base ring has opened at a $\mathrm{C}-\mathrm{N}$ bond due to the shock. In this figure and those that follow, the water molecules are shown with sticks and the nucleotide is depicted using tubes.

the presence of an excess electron, it is important to re- 


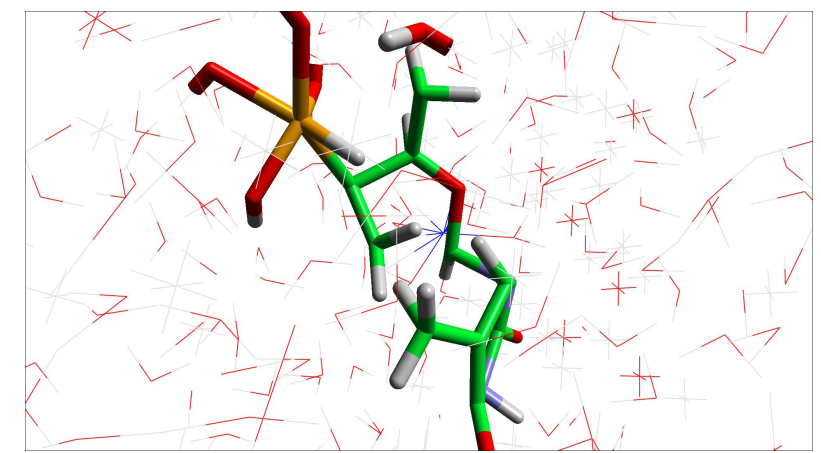

Figure 9. (color online) Nucleotide after a DSW initiated with $V=10 \mathrm{~km} / \mathrm{s}\left(V_{\text {eff }} \approx 4 \mathrm{~km} / \mathrm{s}\right)$, with an excess electron. The phosphodiester $\mathrm{C}-\mathrm{O}$ bond connecting sugar and phosphate broke, and an unusual bond formed between $\mathrm{P}$ and $\mathrm{C}$ in the sugar ring. A $\mathrm{C}-\mathrm{C}$ bond in the sugar ring has opened. This kind of modification is irreversible.

mark that similar situations have been observed in the neutral system. This is not surprising if we take into account the previous observation, that under the action of the SW the geometry of the nuecleotide is so distorted that the excess electron prefers to relocate to the water.

\section{CONCLUDING REMARKS}

The results presented here provide valuable information about the kind of thermo-mechanical processes that are expected when a biological medium is irradiated with swift ions. In general, the phosphodiester bond associated with strand breaks in DNA appears as the weakest link. This dynamical way of producing a break, however, is not always irreversible. In fact, we have repeatedly observed bond reforming provided that the two fragments do not move apart too fast. This is quite feasible given that the compression produced by the SW does not favor shearing deformations.

Within the context of ion irradiation, we observe that the pressure threshold necessary to produce nucleotide damage depends on the ion employed - through its LET
- and on the distance from the ion track to the nucleotide. In the inset to Fig. 6 we plotted pressure vs distance for four different ions: $\mathrm{C}, \mathrm{Ne}, \mathrm{Ar}$ and Fe, with their LET computed according to Eq. (1). If the track is within 5 $\AA$ of the nucleotide, then the pressure generated by the SW will be large enough to produce direct bond breaking independently the ion employed. However, if the distance is larger than $20 \AA$, only the heavier ions like Ar or Fe will produce large enough pressures in the nucleotide region. In particular, protons are very unlikely to produce any thermo-mechanical damage, while $\mathrm{C}$ ions have to pass quite close to the DNA. Note that these conclusions confirm some of the results of Ref. [17].

The analysis of ion-induced biodamage reported in this work, where the interatomic interactions are described at the first-principles level via DFT calculations, provides a valuable methodology capable of generating the necessary inputs for the multiscale scenario linking the irradiation induced complex molecular damages with the biological outcomes of the irradiation $[20,24]$ Our analysis shows that, for light ions like protons or even first-row elements, the SW effect will be insufficient to induce a significant direct damage to biomolecules due to their relatively low LET. They will, however, affect the chemistry and the production of reactive species in the vicinity of the ion tracks quite substantially as it was also recently reported in [27] on the basis of classical MD simulations. For cosmic-ray irradiation involving heavier ions like iron, SW are likely to play a relevant role even for the directly induced damage.

\section{ACKNOWLEDGMENTS}

This work was supported by the COST action MP1002 (Nano-IBCT) through a STSM. The simulations were run in the UK national facility ARCHER, through the UKCP consortium funded by EPSRC grant EP/K013459/1. AFG thanks L. Boüessel du Bourg and E. McBride for help with Aten, DLPOLY and CP2K. We thank A. Yakubovich and E. Bringa for useful discussions and the reviewers for their constructive criticism.
[1] C. von Sonntag, The Chemical Basis of Radiation Biology (Taylor and Francis, London, 1987).

[2] C. von Sonntag, Radical Damage to DNA (Taylor and Francis, London, 1995).

[3] S. Steenken, Chem. Rev. 89, 503 (1989).

[4] W. A. Bernhard in Radical and Radical Ion Reactivity in Nucleic Acid Chemistry, pp. 41-68, ed. M. Greenberg, (Wiley, New York, 2010).

[5] B. Boudaïffa, P. Cloutier, D. Hunting, M. Huels and L. Sanche, Science 287, 1658 (2000).

[6] S. M. Pimblott and J. A. LaVerne, Radiat. Phys. Chem. 76, 1244 (2007).
[7] E. Scifoni, E. Surdutovich and A. V. Solov'yov, Phys. Rev. E 81, 021903 (2010).

[8] M. Smyth and J. Kohanoff, Phys. Rev. Lett. 106, 238108 (2011).

[9] M. Smyth and J. Kohanoff, J. Am. Chem. Soc. 134, 6122 (2012).

[10] Bin Gu, M. Smyth and J. Kohanoff, Phys. Chem. Chem. Phys. 16, 24350 (2014) .

[11] M. Smyth, J. Kohanoff and I. Fabrikant, J. Chem. Phys. 140, 184313 (2014).

[12] M. McAllister, M. Smyth, Bin Gu, G. A. Tribello, and J. Kohanoff, J. Phys. Chem. Lett. 6, 3091 (2015). 
[13] J. Kohanoff, M. McAllister, Bin Gu, and G. A. Tribello, J. Phys. Condens. Matter 29, 383001 (2017), and references therein.

[14] Y. Wu, C. J. Mundy, M. E. Colvin, and R. Car, J. Phys. Chem. A 108, 2922 (2004).

[15] M. Toulemonde, E. Surdutovich and A. V. Solov'yov, Phys. Rev. E 80, 031913 (2009).

[16] E. Surdutovich and A. V. Solov'yov, Phys. Rev. E 82, 051915 (2010).

[17] E. Surdutovich, A. V. Yakubovich, and A. V. Solov'yov, Scientific Reports 3, 1289 (2013).

[18] O. I. Obolensky, E. Surdutovich, I. Pshenichnov, I. Mishustin, A. V. Solov'yov, and W. Greiner, Nucl. Inst. Meth. Phys. Res. B 266, 1623 (2008).

[19] P. de Vera, R. García-Molina, I. Abril, and A.V. Solov'yov, Phys. Rev. Lett. 110, 148104 (2013).

[20] E. Surdutovich and A. V. Solov'yov, Eur. Phys. J. D 68, 353 (2014).

[21] E. Surdutovich and A.V. Solov'yov, Europ. Phys. J. D 69, 193 (2015).

[22] L. Landau and E. Lifshitz, Fluid Dynamics, 2nd ed., Vol. 6 (Reed-Elsevier, Oxford, Boston, Johannesburg, 1987).

[23] Y. Zel'dovich and Y. Raiser, Physics of Shock Waves and High- Temperature Hydrodynamic Phenomena, Vol 1 (Oxford Press, New York, 1966)

[24] A. Verkhovtsev, E. Surdutovich, and A.V. Solov'yov, Scientific Reports 6, 27654 (2016).

[25] P. de Vera, N. J. Mason, F. J. Currell, and A. V. Solov'yov, Eur. Phys. J. D 70, 183 (2016).

[26] P. de Vera, E. Surdutovich, N. J. Mason, and A. V. Solov'yov, Eur. Phys. J. D 71, 281 (2017).

[27] P. de Vera, E. Surdutovich, N. J. Mason, F. J. Currell, and A. V. Solov'yov, Eur. Phys. J. D 72, 147 (2018).

[28] H.A. Bethe et al., Blast wave, Los Alamos Report LA2000, Ch. 2 (Los Alamos, 1947).

[29] G. I. Taylor, Proc. Roy. Soc. Lond. A201, 159 (1950).

[30] L. I. Sedov, Prokl. Mat. Mek. 10, 241 (1946).

[31] J. von Neumann, The point source solution, in Blast wave, eds. K. Fuchs, J.O. Hirschfelder, J.L. Magee, R. Peierls, and J. von Neumann (Los Alamos, 1947).

[32] P. O. K. Krehl, History of shock waves, in Handbook of shock waves, eds G. Ben-Dor, O. Igra, and T. Elperin, Vol 1 (Academic Press, San Diego, 2001); P. O. K. Krehl, History of shock waves, explosions and impact, in A chronological and biographical reference (Springer, Berlin, Heidelberg, 2009).

[33] Y. B. Zel'dovich, J. Exp. Theor. Phys. 12, 389 (1942).

[34] A. V. Yakubovich, E. Surdutovich, A. V. Solov'yov, AIP Conf. Proc. 1344, 230 (2011).

[35] D. Bottländer, C. Mücksch, and H. M. Urbassek, Nucl. Inst. Meth. Phys. Res. B 365, 622. (2015).

[36] A.V. Solov'yov (ed.), Nanoscale Insights into Ion-Beam Cancer Therapy (Springer, Cham, Switzerland (2017).

[37] R. H. Cole, Underwater Explosions (Princeton Univ. Press, N.J., 1948).

[38] R. T. Knapp, J. W. Daily, and F. G. Hammit, Cavitation (McGraw-Hill, New York, 1970).

[39] A. Vogel, W. Lauterborn, and R. Timm, J. Fluid. Mech. 206, 299 (1989).

[40] A. Vogel, W. Hentschel, J. Holzfuss, and W. Lauterborn, Ophthalmology 93, 1257 (1986).

[41] A. J. Coleman and J. E. Saunders, Ultrasonics 31, 759 (1993).

[42] M. Delius, Shock Waves 4, 55 (1994).
[43] A. Vogel, S. Busch, K. Jungnickel, and R. Birngruber, Lasers Surg. Med. 15, 32 (1994).

[44] R. O. Esenaliev, A. A. Oraevsky, V. S. Letokhov, A. A. Karabutov, and T. V. Malinsky, Lasers Surg. Med. 13, 470 (1993).

[45] H. G. David and S. D. Hamann, Trans. Faraday Soc. 55, 72 (1959).

[46] S. D. Hamann and M. Linton, Trans. Faraday Soc. 62, 2234 (1966); ibid 65, 2186 (1969).

[47] S. D. Hamann in Modern Aspects of Electrochemistry, eds. B. E. Conway and J. O. M. Bockris (Plenum, New York, 1974), No. 9, p. 126.

[48] R. Chau, A. C. Mitchell, R. W. Minich and W. J. Nellis. J. Chem. Phys. 114, 1361 (2001).

[49] M. Sprik et al. J. Chem. Phys. 105, 15 (1996).

[50] E. Schwegler, G. Galli, F. Gygi and R. Q. Hood, Phys. Rev. Lett. 87, 265501 (2001).

[51] N. Goldman et al., J. Chem. Phys. 130, 124517 (2009).

[52] M. French, S. Hamel, R. Redmer. Phys. Rev. Lett. 107, 185901, (2011).

[53] T. Ikeda, Y. Katayama, H. Saitoh and K. Aoki, J. Chem. Phys. 132, 121102 (2010).

[54] C. J. Sahle et al., Proc. Natl. Acad. Sci. USA 110, 6301 (2013).

[55] J. G. Blank et al., Orig Life Evol. Biosph. 31, 15 (2001).

[56] Y. Furukawa, T. Sekine, M. Oba, T. Kakegawa, and H. Nakazawa, Nature Geosci. 2, 62 (2009).

[57] N. Goldman, E. J. Reed, L. E. Fried, I.-F. W. Kuo, and A. Maiti, Nature Chem. 2, 949 (2010).

[58] Z. Martins, M. C. Price, N. Goldman, M. A. Sephton, and M. J. Burchell, Nature Geosci. 6 ,1045 (2013).

[59] J. Gu, J. Wang, and J. Leszczynski, J. Am. Chem. Soc. 128, 9322 (2008); J. Nucleic Acids Res. 38, 5280 (2010).

[60] T. G. A. Youngs, J. Comput. Chem. 31, 639 (2009).

[61] W. Smith, C. W. Yong and P. M. Rodger, Mol. Sim. 28, 385 (2002).

[62] W. L. Jorgensen, D. S. Maxwell, and J. Tirado-Rives, J. Am. Chem. Soc. 118, 11225 (1996).

[63] J. VandeVondele, M. Krack, F. Mohamed, M. Parrinello, T. Chassaing, and J. Hutter, Comp. Phys. Comm. 167, 103 (2005); CP2K Developers Group Homepage, http://cp2k.berlios.de/

[64] S. Goedecker, M. Teter, and J. Hutter, Phys. Rev. B, 54, 1703 (1996).

[65] J. P. Perdew, K. Burke and M. Ernzerhof, Phys. Rev. Lett. 77, 3865 (1996).

[66] S. Grimme, J. Comput. Chem. 27, 1787 (2006).

[67] J. Simons, Acc. Chem. Res. 39, 772 (2006).

[68] K. Koshiyama et al., Biophys. J. 91, 2198 (2006).

[69] G. E. Duvall and G. R. Fowles, Shock Waves in High Pressure Physics and Chemistry, ed. R. S. Bradley (Academic, San Diego, 1963), Vol. 2, pp. 209-291.

[70] E. J. Reed, L. E. Fried, and J. D. Joannopoulos, Phys. Rev. Lett. 90, 235503 (2003).

[71] E. J. Reed, L. E. Fried, W. D. Henshaw, and C. M. Tarver, Phys. Rev. E 74, 056706 (2006).

[72] T. R. Mattson and M. P. Desjarlais, Phys. Rev. Lett. 97, 017801 (2006).

[73] V. Rizzi, T. N. Todorov, J. Kohanoff, and A. A. Correa, Phys. Rev. B 93, 024306 (2016).

[74] E. J. Reed, J. Phys. Chem. C 116, 2205 (2012).

[75] R. Ravelo, B. L. Holian, T. C. Germann, and P. S. Lomdahl, Phys. Rev. B 70, 014103 (2004).

[76] Notice that the presence of the nucleotide may change 
the compressibility of the medium at low pressures. It has to be taken into account that the experimental data are for pure water and our simulation cell contains a large molecule (33 atoms) that represents a non-negligible frac- tion of the total number of atoms in the simulation box (843) (4\%).

[77] W. B. Holzapfel, J. Chem. Phys. 50, 4424 (1969).

[78] A. C. Mitchell and W. J. Nellis. J. Chem. Phys. 76, 6273 (1982). 